NUCLEIC ACIDS

\section{No New Interactions}

from our Molecular Biology Correspondent

THE organizers of the Christmas meeting of the British Biophysical Society in London on December 14 and 15, made an prising attempt at the impossible, and failed, in the sense that no general picture ever took shape, and no rules concerning the nature of nucleic acid-protein interactions in different circumstances were formulated. The meeting was therefore no more than a collection of talks falling loosely into three or four groups, though in themselves they maintained a high level of interest.

There were two papers on the interaction of nucleases with their substrates. F. M. Richards (Yale) gave a comparative account of the X-ray structures at atomic resolution of two nucleases and their complexes with inhibitors-his own RNase $\mathbf{S}$ and Cotton's staphylococcal nuclease. In the former case there are now some reasonably well-established ideas about the mechanism, and the interaction of the active centre residues with the nucleotide, involving a considerable displacement of one histidine, can be observed in detail. In staphylococcal nuclease the situation is relatively obscure, and there is a strange dearth of residues generally regarded as catalytically interesting (notably histidine) in contact with the substrate. Two tyrosines must be presumed to be implicated in the binding. Richards pointed out that a very large displacement of a phenolic ring (also found to occur in carboxypeptidase on binding of inhibitor) results in fact from a rotation about one $\mathrm{C}-\mathrm{C}$ bond, which is structurally a very small event. No generai conclusions at all are available about the nature of nucleotide binding sites. G. C. K. Roberts (Cambridge) showed the extraordinary degree of stereochemical detail that can be obtained from high-resolution NMR studies, with particular reference to interaction of pancreatic ribonuclease with inhibitors.

L. Lerman (Vanderbilt) offered evidence of a new form of DNA, the $\psi^{-}$ structure, which appears in solutions containing high concentrations of neutral or acidic polymers together with salts. The conditions were selected to simulate more closely the dielectric environment in vivo. Lerman showed that under critically defined conditions an optical and hydrodynamic transition occurs to a form characterized by compact folding and unusual circular dichroism. Strenuous attempts were made to exclude aggregation effects and association with the added polymers. The unusual spectroscopic properties of DNA in phage heads can in principle be explained by the presence of the $\psi$-conformation. There will be some argument about how well the data can be reconciled with published work by Maestre and others.

The work of $\mathbf{M}$. Nomura (Wisconsin) has provided the most important new results in the study of ribosomes in the past few years. At the meeting, Nomura described the details, with assembly maps, showing the relative roles of different proteins, of the reconstitution of active bacterial $50 \mathrm{~S}$ ribosomes. A new departure in his work concerns the function of the colicin factor that kills $E$. coli by inhibiting ribosomes. Recombination experiments with components from colicin-inactivated and normal cells showed that colicin operates on the ribosomal RNA, not, like other antibiotics, on the proteins, and it turns out that the RNA of the $30 \mathrm{~S}$ particle in fact loses nucleotides from its $3^{\prime}$ end. Such a slightly truncated RNA will combine with proteins to regenerate an inactive particle. It differs from intact RNA in that one protein fails to bind, and it was estab lished that this component (P15) is essential to the function of $30 \mathrm{~S}$ ribo- somes. The essential nature of the $3^{\prime}$ terminus is also consistent with the inactivity of $30 \mathrm{~S}$ ribosomes made with the 16S precursor RNA, which is a little longer at both ends. Both in this and the RNA from colicin-treated cells, a dimethyladenine seems to be missing from from the RNA. Nomura also described the isolation of cold-sensitive mutants, which did not grow at $20^{\circ} \mathrm{C}$, and then containing only partly unfolded ribosomal subunits just as when in vitro the heating step is omitted.

J.-P. Waller (Gif-sur-Yvette) presented results showing that methionyl-tRNA synthetase on proteolysis dissociates from a tetramer to an active dimer, with loss of an 11,000 molecular weight fragment. He suggests that this is, in effect, a separate protein, covalently associated with the rest possibly in consequence of gene fusion, and that it directs the formation of the correct quaternary structure. It is not yet clear why the tetrameric state should be advantageous.

\title{
Sweeping out Double Stranded RNA
}

THE idea that the single stranded RNA genomes of the RNA tumour viruses may be replicated independently of the synthesis of a complementary DNA molecule is rendered very unlikely by the work of K. K. Reddi to be published in next Wednesday's Nature New Biology.

RNA viruses live in a world dominated by DNA; the genetic material of the cells which they parasitize is after all DNA, and as far as is known cellular RNA molecules function only as intermediaries in the expression of DNA genes. The host cell has all the enzymatic machinery required for the self-replication of DNA but presumably it lacks enzymes which can replicate RNA because it has no use for them. The evolution of the RNA viruses has therefore depended on the emergence of some mechanism for the faithful replication of RNA molecules.

On the face of things there are only two alternative pathways for the replication of an RNA genome - either the RNA can be self-replicated quite independently of any DNA metabolism, or the viral RNA genome might be copied into DNA which could then be replicated in the same way as the cell DNA and also act as a template for the synthesis of progeny RNA molecules. Apparently the double stranded RNA viruses and most of the single stranded RNA viruses, including the RNA bacteriophages, polio virus and its relatives, the influenza viruses and the parainfluenza viruses have evolved along the first of these paths. They seem to specify an enzyme or enzymes which can replicate RNA directly. And of course in the case of the single stranded RNA viruses this replication involves the conversion of the input single strand into a double stranded RNA molecule by the synthesis of an RNA complementary to that in the virion which infects the cell.

The RNA tumour viruses and a few other single stranded RNA viruses, by contrast, have evolved along the second path. As Temin and Baltimore proved last year, the replication of these viruses involves the synthesis of a double stranded viral DNA from the input single stranded viral RNA. But might not these viral RNAs be self-replicated as well as replicated by way of a DNA ? There is no reason a priori why this should not happen, and early last year Biswal and Benyesh-Melnick in Texas and Griensven and his colleagues in France claimed to have discovered a double stranded RNA in mouse cells infected and transformed by mouse leukaemia and sarcoma viruses.

Attempts to repeat these observations have, however, failed. K. K. Reddi now reports experiments which drive another nail into the coffin of the idea that RNA tumour viruses can replicate other than by way of a DNA intermediary. In short, Reddi has searched for a double stranded RNA in chick cells transformed by Rous sarcoma virus, taking care to use methods which do not generate artefactually double stranded RNAs. The only double stranded nucleic acid he can detect is DNA, not RNA. As he says, "evidence for the existence of ribonuclease-resistant RNA is lacking", and only double stranded RNA is ribonuclease resistant. Negative results are notoriously difficult to interpret in a meaningful way but, with the odds stacked so high against it, the suggestion that the RNA genomes of RNA tumour viruses can replicate independently of a DNA intermediate is probably best forgotten. 